\title{
Multidimensional Time Model for Probability Cumulative Function Applied to Geometrical Predictions
}

\author{
Michael Fundator \\ Division of Behavioral and Social Sciences and Education, National Academies of Sciences, Engineering, Medicine, Washington D.C., USA
}

Email address:

michaelfundator@gmail.com

To cite this article:

Michael Fundator. Multidimensional Time Model for Probability Cumulative Function Applied to Geometrical Predictions Applied and Computational Mathematics. Vol. 7, No. 3, 2018, pp. 89-93. doi: 10.11648/j.acm.20180703.13

Received: November 9, 2017; Accepted: February 8, 2018; Published: July 5, 2018

\begin{abstract}
Multidimensional Time Model for Probability Cumulative Function can be reduced to finite-dimensional time model, which can be characterized by Boolean algebra for operations over events and their probabilities and index set for reduction of infinite dimensional time model to finite number of dimensions of time model through application of Boolean prime ideal theorem and Stone duality and can be indexed by an index set considering also the fractal-dimensional time arising from alike supersymmetrical properties of probability through consideration of extension of the classical Stone duality to the category of Boolean spaces, locally compact Hausdorff spaces. The introduction of probabilistical prediction philosophically based on Erdős-Rényi LLN for the prediction through Descartes' cycles, Gauss methods of trigonometric interpolation and least squares to reduce error in determination of the orbits of planetary bodies, and Farey series continued by sampling on the Sierpinski gasket.
\end{abstract}

Keywords: Multidimensional Time Model, Law of Large Numbers, Geometrical Predictions

\section{Introduction Discussing Different Approaches to Multidimensional Time Model}

\subsection{First Approach to Multidimensional Time Model Through Kramers Turnover Problem in the Theory of Velocity of Chemical Reactions}

Consider first the mathematical structure of the models of Boltzmann type kinetic equations for reacting gas mixtures for particles undergoing inelastic interactions with reactions of bimolecular and dissociation-recombination type is very complicated, because of the collisional operators that usually in the full Boltzmann equations, are expressed by 5-fold integrals. Consequently direct numerical applications of these models present several computational difficulties. The search for the simpler solution had its long way till the introduction of the equation for the Brownian motion by Albert Einstein. However, using the theory of Brownian motion for the velocity (rate) of chemical reactions Bohr, Kramers, and Slater used only one-dimensional (1D) model for The Kramers turnover problem, that is, obtaining a uniform expression for the rate of escape of a particle over a barrier for any value of the external friction until it was corrected by Grote-Hynes theory 40 years later, with new improvements following, all of them distinguish 1D approach from 2D, 3D, and multiD approaches. First approach to multidimensional time model is through Kramers turnover problem in the theory of velocity of chemical reactions.

It is important and very interesting to consider such point that Kramers in his original work had it as possibility that multidimensional pattern could be related to time dimensions, as he based his introduction theory of Brownian motion on the Einstein's pattern he considered a range of time intervals $\tau$. His discussion of the possibility of a term proportional to $\tau$ in the expression for Moments of Brownian

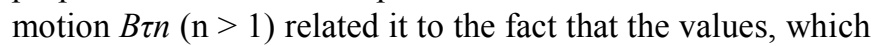
$\mathrm{X}$ takes at moments $\mathrm{t}_{1}, \mathrm{t}_{2} \ldots \mathrm{t}_{\mathrm{n}}$ which lie sufficiently close together are no longer independent; and Moments of Brownian motion Bin $(n>1)$ in fact are represented by a volume integral

$$
\int \ldots \int X\left(\mathrm{t}_{1}\right) X\left(\mathrm{t}_{2}\right) . . X\left(\mathrm{t}_{\mathrm{n}}\right) d \mathrm{t}_{1} d \mathrm{t}_{2} \ldots . d \mathrm{t}_{\mathrm{n}}
$$

over an n-dimensional cube; the contribution to this integral due to a narrow cylinder extending along the diagonal $t_{1}=t_{2}$ 
$=\ldots=\mathrm{t}_{\mathrm{n}}$ may give a term proportional to $\tau$.

\subsection{Second Approach to Multidimensional Time Model Through Cumulant Functions and Time Series Analysis}

To strengthen the above introduced notion consider cumulants properties for time series analysis that provide measure of Gaussianity. If r.v. $X$ is normal, then cumk $\{X\}=0$ for $\mathrm{k}>2$, where cumk denotes the joint cumulants of $\mathrm{X}$ with itself $\mathrm{k}$ times.

For simplicity consider seq of iid $\mathrm{X}_{\mathrm{i}}$ with all moments and $\mathrm{E}\left\{\mathrm{X}_{\mathrm{i}}\right\}=0$ and $\operatorname{var}\left\{\mathrm{X}_{\mathrm{i}}\right\}=1$, then for

$$
\begin{aligned}
\mathrm{Sn}_{\mathrm{n}} & =\Sigma \mathrm{Xi} / \sqrt{n} \\
\operatorname{cumk}\left\{\mathrm{Sn}_{\mathrm{n}}\right\} & =\mathrm{n} \text { cumk }\{\mathrm{X}\} / \mathrm{n}^{\mathrm{k} / 2}
\end{aligned}
$$

that tends to 0 for $\mathrm{k}>2$, as $\mathrm{n}$ tends to infinity, so $\mathrm{S}$ has a limiting normal distribution.

And for time series analysis the moment function

$\mathrm{E}\{\mathrm{X}(\mathrm{t}+\mathrm{u} 1) \ldots \mathrm{X}(\mathrm{t}+\mathrm{uk}-1) \mathrm{X}(\mathrm{t})\}$ would not depend on $\mathrm{t}$, and on the short time interval centered at point of time $t$ can be approximated by normal distribution.

\subsection{Third Approach Through Associated Random Variables}

Additional to the Brownian motion considerations in the theory of chemical reactions and time series analysis for cumulant functions, the same results can be obtained from the consideration of associated random variables.

Definition 1 For $\mathrm{n}>1$ the set of $\mathrm{rv} \mathrm{X}_{\mathrm{i}}$ is said to beassociated, if for all given real-valued functions $g_{i}$ that are increasing ineach component when the other components are held fixed, the inequality

$$
\mathrm{E}[\Pi g j 2 j(\mathrm{X})] \geq \Pi E 2 j(\mathrm{gj}(\mathrm{X})) \text { holds, or equivalently, Corr (gi (X), gj (X)) } \geq 0 \text {, }
$$

Theorem 1. (a) A set consisting of a single random variable is a set of associated random variables. (b) Independent random variables are associated random variables. (c) A subset of a set of associated random variables forms a set of associated random variables. (d) Increasing functions of associated random variables are associated random variables [24].

\section{Multidimensional Time Model for Probability Cumulative Function Is a Method That Opens a New Approach to Geometrical Predictions}

Proposition 1. The process $\mathrm{X}(\mathrm{t})$ with above properties can be represented by composition of Brownian motion processes in finite-dimensional time model.

Next consider Stone representation of Boolean algebra, which is represented by an algebra with known axioms for Boolean algebra and can be characterized by quadruplets $\mathbf{B}=$ $<\mathrm{X}, 0, *, \sim>$, where 0 is an element from a set $\mathrm{X}$, and * is a binary operation and $\sim$ is an unary operation, which would be a Boolean algebra with 1 as a unit on the operations $\wedge, \vee$, and $\sim$. Besides that it has four unary operations, two of which are constant operations, another is the identity, and negation and besides the number of n-ary operations, the number of the dimensions that infinite-dimensional model can be reduced to through application of Boolean prime ideal theorem and Stone duality, can be indexed by an index set.

Proposition 2. Multidimensional Time Model for Probability Cumulative Function can be reduced to finitedimensional time model, which can be characterized by Boolean algebra for operations over events and their probabilities and index set for reduction of infinite dimensional time model to finite number of dimensions of time model considering the fractal-dimensional time that is arising from alike supersymmetrical properties of probability.

\section{Discussion of the History of the Laws of Large Numbers [14-17, 20-22] as a Tricential Method for Predictions}

Statistical prediction in modern sciences traces its roots in ancient mathematics. To introduce this, it would be reasonable to start from the theorems of Probability and Statistics. Such theorems support the calculation of probability, estimation of and testing statistical hypotheses (TSH) for the next occurrence of the Heads and Tails, or $+\mathrm{s}$ and $-\mathrm{s}$. For instance, the theorem of Bernoulli for the probability of coin tossing outcomes of Heads and Tails depending on their frequencies. Consider Erdos-Renyi law of large numbers for general sequences of independent identically distributed (i.i.d.) random variables. As an extension of the Erdos' result that was obtained the same year when Erdos and Selberg found an elementary probabilistical proof of Prime Number Theorem. The theorem extended Kac, Salem, and Zygmund result for functions with conditions

$$
\begin{gathered}
\mathrm{f}(\mathrm{x}+1)=\mathrm{f}(\mathrm{x}), \int_{0}^{1} f(x)=0, \int_{0}^{1} f^{2}(x)=1, \\
\int_{0}^{1}\left(f(x)-\varphi_{n}(f)\right)^{2}=\mathrm{O}\left(\frac{1}{(\log n)^{\epsilon}}\right) \text { for some } \epsilon>0,
\end{gathered}
$$

where $\varphi_{n}(f)$ is the nth partial sum of the Fourier series of $\mathrm{f}$ (x), with $\mathrm{n}_{1}<\mathrm{n}_{2}<\ldots<\mathrm{n}_{\mathrm{k}}<\cdots$ a sequence of numbers satisfying $n_{k+1} / n_{k}>c>1$, then for almost all $x$

$$
\lim _{N \rightarrow \infty} \frac{1}{N}\left(\sum_{k=1}^{N} f\left(n_{k} x\right)=0\right.
$$

to the Theorem of existence of $f(x)$ and sequence $n_{k}$ such that

$$
\limsup _{N \rightarrow \infty} \frac{1}{N}\left(\sum_{k=1}^{N} f\left(n_{k} x\right)=\infty\right.
$$


This result surprisingly points to a philosophical view of prediction at infinity. The question, if the limit in (1) is true for all $\mathrm{f}(\mathrm{x})$ was already proved by Raikov for sequences with $\mathrm{n}_{\mathrm{k}}=2^{\mathrm{k}}$.

And then he further used the gap between the conditions:

$$
\int_{0}^{1}\left(f(x)-\varphi_{n}(f)\right)^{2}=\mathrm{O}\left(\frac{1}{(\log \log n)^{2+\epsilon}}\right),
$$

And

$$
\int_{0}^{1}\left(f(x)-\varphi_{n}(f)\right)^{2}<\frac{1}{(\log \log \log n)^{\epsilon}}
$$

to prove even stronger version

$$
\limsup _{N \rightarrow \infty} \frac{1}{N(\log \log N)^{\frac{1}{2}-\epsilon}}\left(\sum_{k=1}^{N} f\left(n_{k} x\right)\right)=\infty,
$$

And

$$
\limsup _{N \rightarrow \infty} \frac{1}{N(\log N)^{\frac{1}{2}+\epsilon}}\left(\sum_{k=1}^{N} f\left(n_{k} x\right)\right)=0
$$

This result established the weak sense of prediction for the coin tossing outcomes, or the length of the longest heads run that could be used further for the development of method of $\mathrm{TSH}$ from the axiomatical approach to mathematical theory of probability.

The history of application of the law of large numbers (LLN) to statistical analysis started possibly some 450 years ago, when Gerolamo Cardano stated without proof that the accuracies of empirical statistics tend to improve with the number of trials. John Arbuthnot was the first to publish statistical test on fraction of boys and girls born year after year, three years before the LLN for coin tossing random variable was first proved by Jacob Bernoulli. However, more than 120 years passed before Poisson used the name "la loi des grands nombres" ("The law of large numbers"). Five other versions of LLN were derived by Chebyshev, Markov, Borel, Kolmogorov, and Khinchin. They differ from one another by convergence in probability (weak LLN), almost sure convergence, or with probability 1 (strong LLN, e.g. Kolmogorov LLN), if the i.i.d. random variables in the sequence should have a variance (e.g. Kolmogorov LLN), if the variables can be correlated, and the like variations of the LLN. Many of them are proved with the help of Chebyshev inequality.

$$
\mathrm{P}(|-\mu| \geq k \sigma) \leq 1 / \mathrm{k}^{2}
$$

Borel's LLN is the extension of Bernoulli's Theorem that the limiting frequency of the repeating event tends to probability of this event with probability 1 . This is direct consequence of Kolmogorov LLN. Khintchin's weak LLN is the convergence in probability of sample average to the expected value.

$$
\lim _{n \rightarrow \infty} P(|-\mu|>\varepsilon)=0
$$

\section{Insights into Near-Gaussian Distributions and d-Dimensional Projections as a More Than Two Centuries Used Method for Geometrical Predictions}

Before considering any discussion about the above phenomenon or any possible approach to analyze or investigate it, it seems appropriate to quote Karl Pearson, who wrote 110 years ago on p. 189 "My custom of terming the curve the Gauss-Laplacian or normal curve saves us from proportioning the merit of discovery between the two great astronomer mathematicians." One of the definitions of Peirce of "normal" as of what would, in the long run, occur under certain circumstances, clearly implies Principle of prediction and LLN.

"It is undeniable that, in a large number of important applications, we meet distributions which are at least approximately normal. Such is the case, e.g., with the distributions of errors of physical and astronomical measurements, a great number of demographical and biological distributions, etc." Cramer.

The first investigation of slightly non-Gaussian distributions was undertaken by Chebyshev around a century and a half ago, who studied in detail a family of orthogonal polynomials which form a natural basis for the expansions of these distributions. A few years later the same polynomials were also investigated by Hermite and they are called Chebyshev-Hermite or simply Hermite polynomials, their definition was first given by Laplace.

These methods use Edgeworth's form that is equivalent to the Gram-Charlier Type A series with use cumulant analysis for the representation of the distribution function in terms of different types of sums of functions of Gaussian processes.

A standard method of exploring high-dimensional datasets is to examine various low-dimensional projections thereof. In fact, many statistical procedures are based explicitly or implicitly on a projection pursuit. Under weak regularity conditions on a distribution $P=P(n)$ on $\mathrm{R}^{\mathrm{n}}$, most $d$ dimensional orthonormal projections of $P$ are similar (in the weak topology) to a mixture of centered, spherically symmetric Gaussian distributions on $\mathrm{R}^{\mathrm{d}}$ if $\mathrm{n}$ tends to infinity while $\mathrm{d}$ is fixed. [18]

\section{Examples of Geometrical Predictions}

It was established so far that statistical analysis leads to the week sense of prediction, which we call Principle. The Principle of prediction leads to different statistical analyses. There is no surprise why in the further analysis of Laplace's several works on probability, we find that Chapter IV of Laplace's "Philosophical Essay on Probabilities" is called "Concerning hope". The Treatise is one of the important works on the subject of probability where possibly, a half of the Treatise is concerned with statistical methods and applications. In the beginning of Chapter XVII "Concerning 
the various means of approaching certainty", he describes the principles that are components of the Principle of prediction. These principles include induction, analogy and hypotheses, supported by numerous comparisons, which he called "principal means for arriving at truth". In his view analysis and natural philosophy of sciences, Newton's binomial theorem and the principle of universal gravity are the consequences of induction. To show that induction should be followed as far as anticipated with logic which is strengthened by analogy and hypotheses testing, he brings as an example Fermat Conjecture that states that

$2^{2^{n}}+1$ is a prime for all $n$, which was caused by induction and was recognized by Euler that for $n=32$ gives 4,294,967,297, a number divisible by 641 (pp. 177, 178).

In the account of Laplace on the history of the subject, Pascal and Fermat were the first to state the principles and the methods of probability (pp. 167, 185).

In light of the above account about induction and Fermat Conjecture, a reasonable continuation is the Descartes' theorem. Descartes was a very close friend of Fermat and one of the founders of French Academy. The theorem states, that if there are 3 mutual tangent circles to each other, then the $4^{\text {th }}$ circle, which can possibly be circumscribed or inscribed could be constructed. The $4^{\text {th }}$ circle would be tangent to all 3 circles and the 4 th curvature could be calculated from the first 3 from equation:

$$
\left(\mathrm{k}_{1}+\mathrm{k}_{2}+\mathrm{k}_{3}+\mathrm{k}_{4}\right)^{2}=2\left(\mathrm{k}_{1}^{2}+\mathrm{k}_{2}^{2}+\mathrm{k}_{3}^{2}+\mathrm{k}_{4}^{2}\right), \mathrm{k}_{\mathrm{i}}= \pm 1 / \mathrm{r}_{\mathrm{i}}
$$

with $r_{i}$ being radius of the $i$-th circle

Euler showed that the special case, when the $\mathrm{k}_{\mathrm{i}}$ are perfect squares of $a_{i}^{2}$, is equivalent to the three simultaneous equations of Pythagorean triples.

$$
\begin{aligned}
& \left(a_{1}^{2}+a_{2}^{2}+a_{3}^{2}+a_{4}^{2}\right)^{2}=2\left(a_{1}^{4}+a_{2}^{4}+a_{3}^{4}+a_{4}^{4}\right) \\
& 2\left(a_{1} a_{2}\right)^{2}+2\left(a_{3} a_{4}\right)^{2}=\left(a_{1}^{2}+a_{2}^{2}-a_{3}^{2}-a_{4}^{2}\right)^{2} \\
& 2\left(a_{1} a_{3}\right)^{2}+2\left(a_{2} a_{4}\right)^{2}=\left(a_{4}^{2}-a_{2}^{2}+a_{3}^{2}-a_{4}^{2}\right)^{2} \\
& 2\left(a_{1} a_{4}\right)^{2}+2\left(a_{2} a_{3}\right)^{2}=\left(a_{1}^{2}-a_{2}^{2}-a_{3}^{2}+a_{4}^{2}\right)^{2}
\end{aligned}
$$

It is also interesting to notice that Fermat, who was in one Mersenne circle with Descartes, and later in exchanged correspondence with Pascal laid foundations of the theory of probability, gave the smallest Pythagorean triple with both the hypotenuse $\mathrm{c}$ and the sum of the sides $\mathrm{a}+\mathrm{b}$ as perfect squares such triple has sides

$$
\mathrm{a}=4,565,486,027,761 ; \mathrm{b}=1,061,652,293,520 ; \text { and } \mathrm{c}=4,687,298,610,289,
$$

with $\mathrm{a}+\mathrm{b}=2,372,159^{2}$ and $\mathrm{c}=2,165,017^{2}$, although there are infinitely many such Pythagorean triples.

It is also interesting to notice that there is a general formula that gives all solutions of Fermat cubic

$$
\begin{gathered}
a_{1}^{3}+a_{2}^{3}+a_{3}^{3}=a_{4}^{3}, \\
\left(3 x^{2}+5 x y-5 y^{2}\right)^{3}+\left(4 x^{2}-4 x y+6 y^{2}\right)^{3}+\left(5 x^{2}-5 x y-3 y^{2}\right)^{3}=\left(6 x^{2}-4 x y+4 y^{2}\right)^{3}
\end{gathered}
$$

Farey sequence that was first studied by C. Haros a year after Gauss' successful calculation of the orbit of Ceres was introduced 200 years ago. It can be viewed as a natural continuation of Descartes' circles theorem, after more than 120 years, when L. R. Ford presented a very important property of Farey's series. The property indicates that circles of radius $1 / \mathrm{b}^{2}$ drawn above each reduced fraction $a / b$, and touching the number line at this point never overlap despite expectations, although they touch very often. It was first studied by C. Haros a year after Gauss' successful calculation of the orbit of Ceres, and thus was introduced 200 years ago with Farey's paper.

\section{Conclusion}

With the introduction of Descartes' Circle Theorem in his letter to Princess Elisabeth of the Palatinate it is almost a quarter of a century less from Quadricentennial history of Geometrical Predictions. The Theorem was rediscovered by Philip Beecroft almost 200 years later and then by Frederick Soddy almost 100 years later. This makes the quadricentennial history of Geometrical Predictions look like Tercentennial history of the Law of Large Numbers that started with publication of Bernoulli's Theorem and had its Centenary and Bicentenary anniversaries commemorated by Laplace's publication of his Treatise on Probability, translations of Ars Conjectandi and publications on the history of the LLN for the Bicentenary celebrations The Tricentenary that is also the 250th

anniversary of the first public presentation of Thomas Bayes's work was commemorated in Paris in at a colloquium entitled L'art de conjecturer des Bernoulli [10-12, 20-21].

\section{References}

[1] M. Fundator Applications of Multidimensional Time Model for Probability Cumulative Function for Parameter and Risk Reduction. In JSM Proceedings Health Policy Statistics Section Alexandria, VA: American Statistical Association. 433-441.

[2] M. Fundator. Multidimensional Time Model for Probability Cumulative Function. In JSM Proceedings Health Policy Statistics Section. 4029-4039.

[3] M. Fundator. Testing Statistical Hypothesis in Light of Mathematical Aspects in Analysis of Probability doi:10.20944/preprints201607.0069.v1.

[4] M. Fundator Application of Multidimensional time model for probability Cumulative Function to Brownian motion on fractals in chemical reactions (44th Middle Atlantic Regional Meeting, June/9-12/16, Riverdale, NY) Academia Journal of Scientific Research (ISSN 2315-7712) DOI: 10.15413/ajsr.2016.0167 In preparation for publication. 
[5] Michael Fundator Application of Multidimensional time model for probability Cumulative Function to Brownian motion on fractals in chemical reactions (Northeast Regional Meeting, Binghamton, NY, October/5-8/16). Academia Journal of Scientific Research (ISSN 2315-7712) DOI: 10.15413/ajsr.2016.0168 In preparation for publication.

[6] Michael Fundator Applications of Multidimensional Time Model for Probability Cumulative Function for design and analysis of stepped wedge randomized trials. Academia Journal of Scientific Research (ISSN 2315-7712) DOI: 10.15413/ajsr.2016.0169 In preparation for publication.

[7] Michael Fundator Multidimensional Time Model for Probability Cumulative Function and Connections Between Deterministic Computations and Probabilities Journal of Mathematics and System Science 7 (2017) 101-109 doi: 10.17265/2159-5291/2017.04.001.

[8] A. De Moivre, "The Doctrine of Chances", 2nd ed. (London, England: H. Woodfall, 1738).

[9] Pierre Simon, Marquis De Laplace "Analytical theory of Probability".

[10] Pierre Simon, Marquis De Laplace "Philosophical essay on Probability".

[11] Peirce, Charles S. (c. 1909 MS), Collected Papers.
[12] P. Erdos "On the strong Law of Large Numbers".

[13] Josef Steinebach "On a necessary condition for the ErdosRenyi law of large numbers" Proceedings of the American Mathematical Society Volume 68, Number 1, January/78.

[14] P. Erdös and A. Rényi, On a new law of large numbers, J. Anal. Math. 23 (1970), 103-111.

[15] Sandor Csorgo Erdos-Renyi Laws The Annals of Statistics Vol. 7, No. 4 (Jul., 1979), pp. 772-787.

[16] L. Dumbgen, P. D. Conte-Zerial On low-dimensional projections of high-dimensional distributions.

[17] H. A. Kramers, Brownian motion in a field of force and the diffusion model of chemical reactions. Physica, 7, 4, 284-304 (1940).

[18] Seneta E. A Tricentenary history of the Law of Large Numbers. Bernoulli 19 (4), 2013, 1088-1121.

[19] W. Feller, An Introduction to Probability Theory and its Applications, Vol. 1 (Wiley, New York, 1966).

[20] H S M Coxeter An Absolute Property of Four Mutually Tangent Circles www.math.yorku.ca/dcoxeter/chap $1 / \mathrm{b} \% 20$ $\% 20 \mathrm{An} \% 20$ Absolute $\% 20$ Property.pdf.

[21] Pedoe, Daniel. "On a Theorem in Geometry." Amer. Math. Monthly 74.6 (1967): 627-640. 